\title{
Leading Transformation in Healthcare and the Role of Emotional Intelligence
}

\section{Jacqueline A Hinds*}

Society of Emotional Intelligence, UK

"Corresponding author: Jacqueline A Hinds, Chair, Society of Emotional Intelligence, UK, Tel: +447545018756; E-mail: wilsonhindsconsulting@gmail.com

Received date: April 11, 2017; Accepted date: August 28, 2017; Published date: August 31, 2017

Copyright: () 2017 Hinds JA. This is an open-access article distributed under the terms of the Creative Commons Attribution License, which permits unrestricted use, distribution, and reproduction in any medium, provided the original author and source are credited.

\section{Commentary}

Leading transformation in healthcare has tremendous advantages in improving the services, patient experience and care within the National Health Service (NHS). It also bears the scars and, has had a significant impact on staff undergoing the transformation process and, in a large majority of cases, impairing their duty of care to the patients and services under their care. The concept of Emotional Intelligence (EI) in healthcare, although not referred to or identified as an integral part of the healthcare infrastructure, is in reality, interwoven into everything that is delivered as part of a service provision. In fact, EI is a crucial concept to integrate within healthcare organisations; across all levels of employees and not just apportioned to senior managers or leaders. The knowledge of EI enables individuals to understand their emotions, emotional meanings and to reflectively regulate these emotions whilst transitioning through change initiatives and periods of significant and, sometimes, rapid transformation.

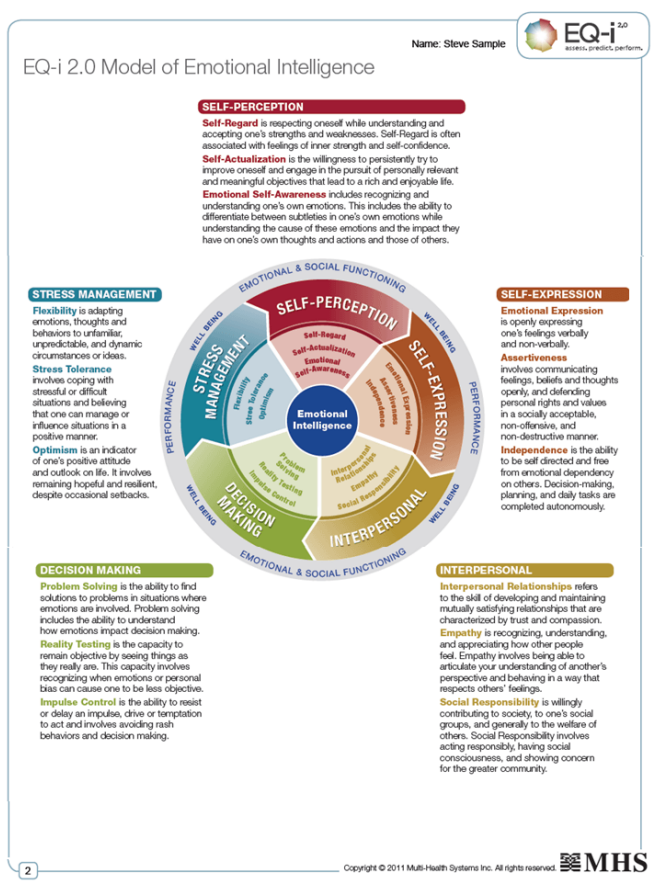

Figure 1: The EQ-i 2.0 Model (1-5-15).

Not all healthcare transformational projects have been successful in the past. Some have been more transactional and results driven, which has led to employees feeling pressured and disturbed about the changes that were taking place and, more often than not, being emotionally unstable or stressed as a result of the changes at that juncture. The link between EI and patient-centered outcomes has been explored by Birks and Watt [1].

Building on their work, I have gleaned insight and experiences within two of the largest merged healthcare organisations in the UK and Europe, which has brought forth a cornucopia of knowledge and experience around leading transformational change in the healthcare and the role of emotional intelligence. Experiences in effective transformational change and, the fallout of transactional change initiatives has over the years, resulted in a significant rise in organisations requesting external consultants to deliver conflict resolution and more recently, anger management training within their establishments.

\section{What is Emotional Intelligence vs. Emotional Quotient?}

EQ and EI are more often than not classed as one and the same, when in fact they are very different. Emotional Quotient (EQ) is a measure of a person's adequacy in areas such as self-perception, empathy and decision making; whereas Emotional Intelligence is about managing one's emotions and that of others' emotions around them.

Emotional Intelligence (EI) is in everything that we do. The term 'Emotional Intelligence' was created by Peter Salovey and Jack Mayer; here they describe the term as an ability to perceive emotions, to access and generate emotions so as to assist thought to understand emotions and emotional meanings, and to reflectively regulate emotions in ways that promote emotional and intellectual growth.

EI is a set of emotional and social skills that collectively establish how well we as individuals not only perceive and express ourselves but how we engage, develop and maintain social and personal relationships, how we overcome or cope with challenges that we may face and, how we use emotional information in an effective and, most meaningful way. Clemons [2] explores how EI could be holding individuals back not only within their personal lives, but within their professional lives too.

"Emotional Intelligence is being 'smart' about our emotions and those of others [3]."

\section{$\sim$ Hank Clemons PhD [2]}

Emotional Quotient (EQ) is how one measures Emotional Intelligence. Mayer et al. [4] state in development of the EQ-i 2.0 model, EI had been defined as a set of emotional and socials skills influencing what is perceived and expressed in ourselves. The model outlined below in Figure 1, depicts EI at the core or centre of the 
model, the composite scales are the high-level categories that contain the subscales; all of which work together to impact emotional and social functioning and overall performance which includes wellbeing or Happiness.

\section{Linking Emotional Intelligence and Transformation}

Mark [3] opens up much debate on how the healthcare arena has explored EI and the impact that it has had on patient-centered care. This lead onto a quest to highlight where Emotional Intelligence has indeed made a significant contribution to organisational change and as a result, leading onto smoother transformation initiatives within the healthcare arena.

Birks and Watt [1] state that studies have begun to examine relationships between EI and stress and burnout in individuals, and that such problems occur within the context of the health care organisations. A wider approach to this arena may need to look closer and examine the organisational culture in which health care is delivered and, whether an organisation can operate in an emotionally intelligent way in order to reduce stress and burnout of healthcare givers. Stress and burnout is a big player in any form of change initiative.

This lack of emotional intelligence is apparent when organisations fail to understand the impact of the decision to embark on merging not only the workforce but, the entire organisational infrastructure. They neglect to comprehend how combining individual healthcare organisations with their own culture, infrastructure and workforce to create one whole new healthcare organisation with new visions and values, structures, combined workforces and skillsets will affect the people.

Moves of this nature are quite significant and to avoid any detrimental impact to the overall patient experience, organisations must realise the emotional pull or overall influence of such change, a merger of any sized organisation in any industry arena, will evoke a range of emotions that will emit distress signals from all areas; sending shockwaves throughout the nervous system of the organisational infrastructure.

This in itself will inadvertently cause stress anxiety and depression across the merged workforce as, not all staff will be adept to any form of change, and will feel even more angst if their job roles and location site are part of the change initiative.

"The NHS in the UK is acutely aware of the role of Emotional Intelligence, especially in medical outcomes and organisational effectiveness."

\section{Daniel Goleman, Author, Emotional Intelligence}

McNulty and Ferlie [5] argued that organisational transformation brought about sedimented rather than transformational change and the limitations of radical top-down change strategies in professionalised public service organisations [6]. Figure 2, outlines a transformational process within a National Healthcare Trust (NHS) in the North of England.

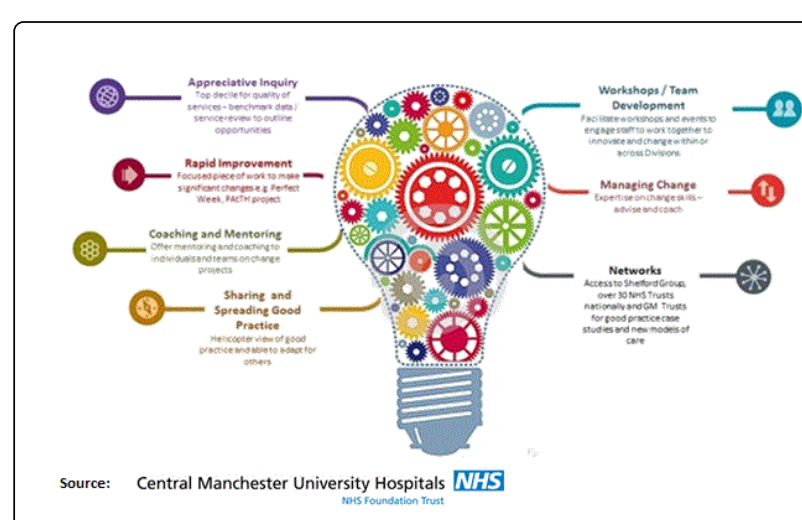

Figure 2: What does transformation look like in healthcare?

The example outlines the Trusts' organisational change initiatives, the key areas for transformation, how they would undertake the transformational process and with whom they will engage with to undertake the process. More often than not, organisations will enlist an external body to undertake high level transformational initiatives; which may or may not involve qualified and competent staff within the organisation. This is purely dependent upon what has been agreed at a higher level and how involved internal consultants will be with the overall change initiative. Each healthcare Trust will focus on their own key transformational initiatives which they believe are essential to organisational change and growth.

Two of the largest merged healthcare organisations were not exempt from the fallout of merging. It was felt that the larger the merged organisation, the more challenges were faced with communication across all sites and throughout all levels of the organisation. Communication channels were somewhat impaired with challenges in disseminating key information on focus groups, training and essential information pertaining to the newly merged or proposed merger.

\section{Imperial College Healthcare NHS Trust}

- $\quad$ Trust formed in October 2007

- Hammersmith Hospitals NHS Trust, St Mary's NHS Trust and Imperial College Faculty of Medicine.

- Managing: Charing Cross, Hammersmith, Queen Charlotte's and Chelsea, St Mary's and Western Eye Hospitals.

- One of the largest Trusts in England circa. 10,000 staff.

\section{Barts Health NHS Trust}

- $\quad$ Trust formed in October 2012.

- Whipps Cross University Hospital, Newham University Hospital, Barts and The London NHS Trust.

- Managing: Barts Hospital, The Royal London Hospital, The London Chest Hospital and Mile End Hospital.

- The largest Trust in England and Europe circa. 15,000 staff.

My experience within both organisations within a Human Resource Development Specialism. enabled a very much hands-on approach to working with staff undergoing transformational change, but in doing so, it somewhat highlighted the flaws and strong need for Emotional 
Intelligence in not only disseminating key information but, in the up skill of people managers and team leaders as well. It was very apparent with the changes in organisational infrastructure and in job roles for individuals, that they were ill-equipped to undertake the role they were promoted to and, this resulted in a short period of time after the merger had taken place, complaints of bullying and harassment, stress, anxiety, sickness and absenteeism and general low staff morale began to filter through at a rapid rate.

As part of the pre and post-merged organisation, a series of training and transitioning programmes were implemented and delivered across the organisations at all key sites; the training targeted all levels of staff transitioning within and outside of the organisation. A suite of offerings ranging from service improvement training for clinical and non-clinical staff, personal and career development and EI coaching was implemented as part of a wholistic intervention; preparing and equipping staff who were predominantly transitioning across the organisation into other roles as a result of departmental restructuring.

Birks and Watt [1] argue that there was no definitive evidence linking Emotional Intelligence to organisational performance. I disagree with this argument. Working in two of the largest merged NHS trusts in UK and Europe, has given me first hand insight into transformational change across all levels of staff within an organisation. Understanding the positive and negative impact an organisation-wide change initiative can bring, gave me the opportunity to tap into my own EI in order to undertake and deploy a range of programmes to suit the situation at hand and; the glaringly obvious need for staff to essentially up skill and transition into the newly merged organisation.

It is not uncommon for essential and expensive career development, training and up skill to remain available only for the higher echelons of the organisational infrastructure. In 2014, Daniel Goleman (well renowned author and scientific journalist) Emotional Intelligence expert and world best-selling author of his book Emotional Intelligence, visited the NHS to speak their Top Leaders on the role of Emotional Intelligence in Healthcare and specifically with medical outcomes. What would it cost to share this essential knowledge to both clinical and non-clinical staff who is working avidly to ensure that everything they do within their roles is to help the patient experience within any healthcare setting?

Can you imagine if this kind of development was brought into an organisation and, was available for all staff and, disseminated in digestible chunks within an organisation that is undergoing transformational change in one form or another? How EI savvy would that make them once they were up skilled?

\section{So what does Emotional Intelligence Look like within Healthcare?}

Figure 3 outlines a similar transformational model to that of the example outlined in Figure 2. The transformation model has a wholistic approach that merges it with EI composite scales and subscales. By incorporating the EQ model and programmes of choice and, by ensuring that all staff with a recruitment and selection aspect to their role as well as HRD and change management staff are fully trained to disseminate the key principles of Emotional Intelligence to staff through coaching, workshops, online assessments and feedback, will create a uniformed approach and smoother transitioning process due to skilled staff who are able to disseminate, develop and lead staff into the Trusts' transformational initiatives.

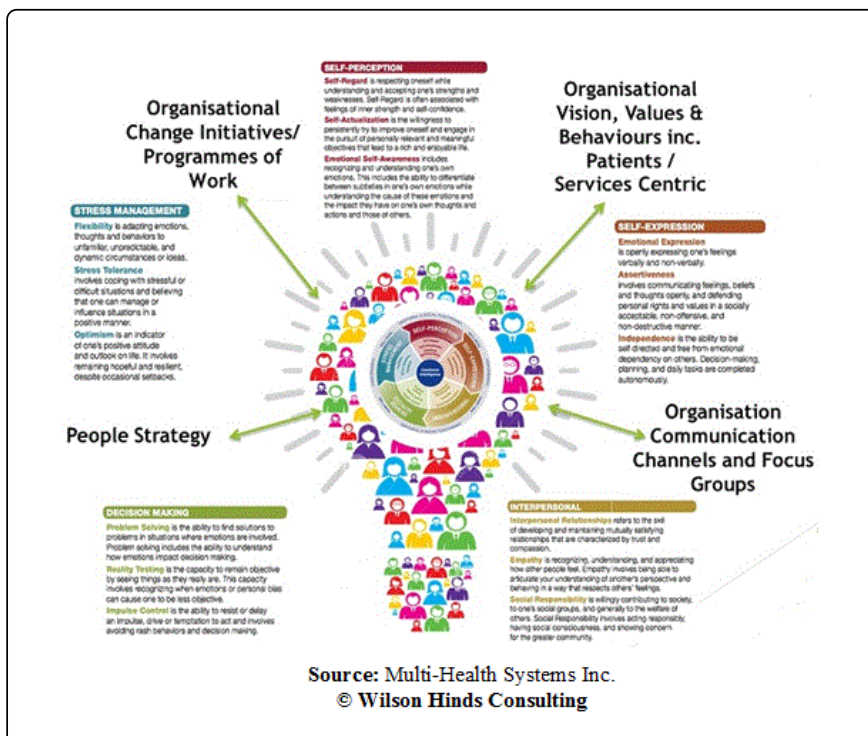

Figure 3: Transformational change-EI model.

\section{What's the Way Forward?}

A big mistake made by organisations is not seeing all our staff as worthy assets, able to deliver on the vision and values that are held up high for all to see. By tapping into your Emotional Intelligence (EI) organisations can empower everyone, from leaders, managers and staff at all levels and within all environments? By understanding their ability and capacity as professionals, leaders can help their staff reach their full potential to not only perceive, assess and manage their emotions, but that of others as well; be it leader, peer or subordinate; this would be deemed a core strength within itself.

Out of this changed culture and transformation process, will arise a new vision of professional healthcare, corporate or public sector practice; embedding EI as a core component within their mandatory compliance infrastructure. Emotionally intelligent staff, means a healthier organisation which for the NHS, can lead to better patient centred-care.

\section{References}

1. Birks YF, Watt IS (2007) Emotional intelligence and patient-centred care. J R Soc Med 100: 8.

2. Clemons H (2012) Could your emotional intelligence be holding you back? Booklocker.com, Inc.

3. Mark A (2005) Organizing Emotions in Healthcare. J Health Organ Manag 19: 277-285.

4. Mayer JD, Salovey P, Caruso D (2002) Mayer-Salovey-Caruso Emotional Intelligence Tests (MSCEIT). User Manual Toronto: Multi-Health Systems Inc.

5. McNulty T, Ferlie E (2004) Process transformation: Limitations to radical organizational change within public service organizations. Organization Studies 25: 8.

6. Stein SJ (2011) The EQ Edge, Third edition: Emotional intelligence and your success. John Wiley \& Sons Canada Ltd. 